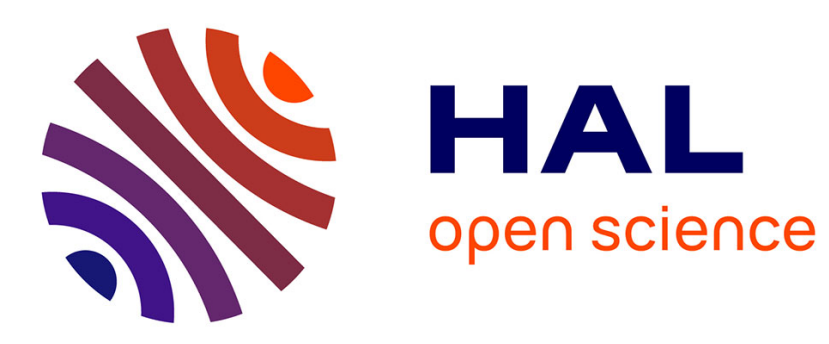

\title{
L'idée d'ordre spontané ou le monde selon Hayek
}

Michel Bourdeau

\section{To cite this version:}

Michel Bourdeau. L'idée d'ordre spontané ou le monde selon Hayek. Archives de Philosophie, 2014, 77 (4), pp.663-678. halshs-01100155

\section{HAL Id: halshs-01100155 https://shs.hal.science/halshs-01100155}

Submitted on 8 Jan 2015

HAL is a multi-disciplinary open access archive for the deposit and dissemination of scientific research documents, whether they are published or not. The documents may come from teaching and research institutions in France or abroad, or from public or private research centers.
L'archive ouverte pluridisciplinaire HAL, est destinée au dépôt et à la diffusion de documents scientifiques de niveau recherche, publiés ou non, émanant des établissements d'enseignement et de recherche français ou étrangers, des laboratoires publics ou privés. 


\title{
L'idée d'ordre spontané ou le monde selon Hayek.
}

\author{
Michel Bourdeau \\ (IHPST, CNRS-Paris1-ENS))
}

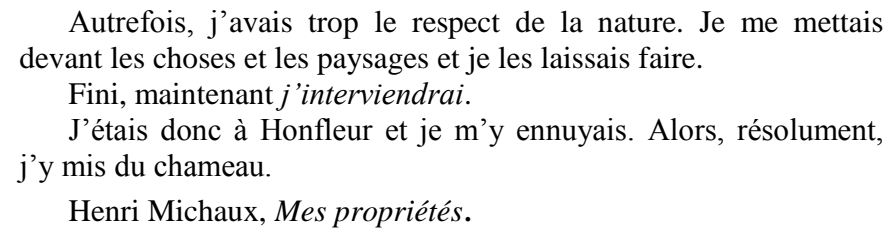

Résumé. L'idée d'ordre spontané occupe chez Hayek une place centrale, puisqu'il en fait le fondement à la fois de la science sociale et du libéralisme. Une première partie rappelle qu'il s'agit d'abord d'une notion épistémologique, nécessaire là où les phénomènes deviennent trop complexes pour qu'on puisse y dégager des lois. La seconde partie, qui traite de la théorie de la catallaxie, ou ordre spontané du marché, est centrée sur la notion de concurrence, conçue comme une procédure de découverte apportant une solution au problème posé par la division du savoir et assurant une coordination sans coordinateur. La dernière partie, qui examine les différents types d'intervention, permis ou proscrits, souligne combien le bon fonctionnement de cet ordre spontané dépend en fait de conditions juridiques qu'il est demandé à l'Etat d'imposer. La conclusion signale deux aspects problématiques de cette théorie.

Mots clés : Hayek, ordre spontané, ordre modifiable, catallaxie, concurrence, coordination sociale.

L'idée d'ordre spontané est ce qui se cache derrière l'image de la main invisible, comme la pulpe sous l'écorce. Vu le rapport immédiat avec la téléologie, l'action de cette main invisible a pu être interprétée en termes théologiques. Dans les philosophies de l'histoire de Kant ou de Hegel, elle apparaît comme une laïcisation de la providence, une métamorphose de la théodicée, mais la théorie darwinienne de l'évolution nous a montré comment faire l'économie de la finalité : comme Hayek, à la suite de bien d'autres, se plaît à le rappeler, l'ordre social spontané est le résultat de l'action humaine, mais non d'un dessein humain.

Parmi les traits distinctifs de la pensée de celui-ci, un des plus remarquables est la place primordiale qu'il accorde à l'idée d'ordre au sein d'une philosophie des sciences. Alors que la plupart de nos contemporains n'y voit qu'une notion politique, le concept possède également à ses yeux une dimension épistémologique, étroitement liée à l'existence de la complexité. C'est là où les phénomènes sont trop complexes pour qu'on puisse en rendre compte à l'aide de lois, en biologie par exemple, que le concept d'ordre se révèle utile. Quant à l'idée d'ordre spontané, c'est-à-dire d'un ordre qui résulte de l'action humaine sans avoir pour autant été voulu, elle est doublement fondamentale puisque, non contente de servir de fondement à la science sociale, elle marque également l'acte de naissance du libéralisme économique. S'il est vrai que celui-ci ne se confond pas avec le simple «laissez-faire ", il n'en reste pas moins qu'à ses yeux, les seules interventions légitimes dans ce domaine doivent exclusivement viser à ce que rien ne vienne entraver le libre fonctionnement des forces ordonnatrices. Sans doute, l'ordre qui en résulte n'est 
pas parfait, mais il serait à la fois vain et dangereux de chercher à exercer sur les phénomènes un contrôle conscient et délibéré, dans l'idée d'obtenir un résultat plus satisfaisant.

Pour exposer l'idée d'ordre spontané telle qu'elle se trouve chez Hayek, je procéderai en trois temps. Une première partie considérera la notion d'ordre en elle-même, en tant qu'elle relève d'une philosophie des sciences, et soulignera plus particulièrement ses liens avec la notion de complexité. Pour étudier l'ordre spontané du marché, encore appelé catallaxie, la seconde partie commencera par rappeler le rôle crucial que jouent les considérations cognitives en économie ; il sera alors possible de montrer comment la concurrence, prise comme procédure de découverte, permet de résoudre, sur le cas particulier du marché, le problème général posé par la coordination, phénomène qui se trouve au cœur de toute science sociale. Une troisième partie s'interrogera alors sur les différentes façons, légitimes ou au contraire néfastes, d'intervenir dans un ordre spontané, mettant en évidence l'intime solidarité existant entre droit et catallaxie : sans mesures légales adéquates, il serait impossible de garantir la bonne marche de l'ordre spontané. Une dernière partie s'interrogera brièvement sur la pertinence de ces analyses.

\section{Une notion épistémologique.}

Ordre et complexité. Contrairement à ce que l'on pourrait croire, la principale raison de s'intéresser à l'idée d'ordre n'est pas à chercher du côté de la politique mais du côté des sciences. Le fait peut paraître surprenant dans la mesure où, hormis en mathématique, les savants ne semblent guère se soucier d'ordre. Au constat dressé par Comte, « la notion des lois naturelles [...] entraîne aussitôt l'idée correspondante d'un certain ordre spontané », fait cependant écho celui de Hayek, «L'ordre est un concept indispensable pour étudier tous les phénomènes complexes, vis-à-vis desquels il doit largement jouer le rôle que le concept de loi joue dans l'analyse des phénomènes plus simples », qui ajoute en note : «ce fut en fait d'abord entièrement en vertu de considérations méthodologiques que je fus conduit à reprendre l'usage du concept impopulaire d' 'ordre' ». En effet, plus les phénomènes deviennent complexes, moins les schémas empruntés à la physique trouvent à s'appliquer. La mesure, la mise en forme mathématique se heurtent à des difficultés jusqu'alors absentes. Quant au pouvoir prédictif de la théorie, Hayek résume la situation en ces termes: «aucun économiste n'a encore réussi à faire fortune en achetant et vendant des marchandises en fonction de ses prédictions scientifiques des prix futurs (bien que certains aient pu faire fortune en vendant de telles prédictions) » (SPPE, p. 74). Il faut donc le plus souvent se contenter de dégager un ordre, c'est-à-dire :

«un état de choses dans lequel une multiplicité d'éléments de nature différente sont en un tel

rapport les uns aux autres que nous puissions apprendre, en connaissant certaines composantes spatiales ou temporelles de l'ensemble, à former des pronostics corrects concernant le reste, ou du moins des pronostics ayant une bonne chance d'être corrects » (DLL, p. 121).

Si l'action de l'aimant sur la limaille de fer nous donne déjà un premier exemple de ces ordres complexes, c'est avec la biologie qu'ils nous sont devenus vraiment familiers. Hayek cite d'autant plus volontiers des travaux comme ceux de Von Bertallanffy qu'il existe une parenté entre la notion d'ordre et celles de système ou de structure. Une fois renoncé à la finalité, il faut bien attribuer l'état d'un organisme à l'auto-organisation et les phénomènes biologiques nous donnent de nombreux exemples de cette auto régulation dont Hayek affirme qu'ils se retrouvent dans le fonctionnement du marché. L'intérêt soutenu de Hayek pour la biologie tient aussi à l'approche génétique qu'il emprunte à ses maîtres autrichiens ; d'où, par le biais de l'évolution culturelle, des rapprochements fréquents avec la théorie darwinienne : évolution et ordre spontané sont deux «idées jumelles » (SPPE, p. 132), la tâche fondamentale de la science sociale comme de la biologie étant d'apporter, chacune dans son domaine, la réponse à une même question : comment rendre compte de la genèse des ordres spontanés? (SSS, p. 136 n 74)

Parmi les phénomènes sociaux, l'exemple le plus clair est fourni par le langage. La grammaire vise à dégager des régularités observées dans le comportement langagier : les structures qu'elle décrit sont souvent d'une grande complexité, elles sont abstraites en ce sens qu'elles échappent à la 
conscience du sujet parlant, et elles changent sans que personne ne cherche à les faire changer. Malgré les apparences, des régularités statistiques comme le sex-ratio ou le nombre des suicides ne constituent pas en revanche d'authentiques ordres spontanés. La statistique participe du préjugé scientiste, objectiviste dont il sera question un peu plus bas : elle prend les phénomènes sociaux « de l'extérieur », ignore la manière dont les éléments dont elle traite sont structurés, de sorte que « les structures qui intéressent les sciences sociales disparaissent en fait » (SSS, p. 68 ; cf. SPPE, p. 66). Dès ce stade, il est donc déjà acquis que, pour des raisons strictement épistémologiques, une science de la société a besoin du concept d'ordre social .

Kosmos et Taxis. Les exemples ci-dessus relèvent majoritairement d'un type d'ordre particulier. L'ordre de bataille d'une armée prête à engager le combat n'a rien de spontané : elle relève de la décision du commandant en chef. Il importe donc de reconnaître que le concept d'ordre recèle une grande équivoque. L'ordre peut en effet être endogène ou exogène. L'ordre dans lequel se déroulent les opérations d'une machine n'a pas son principe en elle, mais dans l'esprit de celui qui l'a conçu, alors que l'ordre dans lequel se déroulent les opérations analogues d'un organisme a son principe dans ce dernier. Dans un cas, l'ordre est un arrangement : chaque chose est à sa place, c'est-à-dire à la place que l'ordonnateur lui a assignée. Dans l'autre cas, il n'y a pas d'ordonnateur, mais simplement des forces ordonnatrices ; l'ordre est auto-généré, spontané.

Pour mettre en valeur cette opposition, Hayek a proposé d'appeler Taxis l'ordre arrangé et Kosmos l'ordre spontané. Trois grands traits les opposent. L'ordre arrangé (Hayek parlera encore d'organisation dans le cas des phénomènes sociaux) est simple, l'ordre spontané est complexe : dans un cas, la disposition des pièces sur l'échiquier, dans l'autre la figure formée par la limaille de fer sous l'action de l'aimant. L'ordre arrangé est concret, immédiatement perceptible, alors que l'ordre spontané est le plus souvent abstrait et passe donc facilement inaperçu. Enfin, l'ordre arrangé résulte d'une intention délibérée, alors que l'ordre spontané se produit sans avoir été voulu comme tel.

Des trois propriétés signalées, la plus importante est la dernière. Si une des «thèses majeures » de Hayek pose que «des ordres extrêmement complexes, comprenant plus de faits distincts qu'aucun cerveau n'en peut constater ou manipuler, ne peuvent être produits qu'à travers des forces poussant à la formation d'ordres spontanés " (DLL, p. 127), il n'en existe pas moins des ordres spontanés qui sont simples. En revanche, un ordre spontané est par définition non intentionnel, puisqu'est dit spontané ce qui ne procède ni d'une réflexion ni d'une contrainte extérieure, mais surgit de lui-même et comme de la nature de la chose. - Cette même propriété explique encore que la confusion dénoncée par Hayek ait tant duré. L'idée que, dans la vie sociale, l'ordre puisse résulter de la seule spontanéité, sans qu'il soit nécessaire de faire appel à une quelconque intention, qu'il puisse y avoir un ordre sans volonté ordonnatrice, a en effet quelque chose de déroutant et notre esprit semble répugner à lui faire une place. Or c'est bien de cela qu'il s'agit: Il n'y a plus de volonté, d'intention ordonnatrice, pas d'ordonnateur mais seulement des forces ordonnatrices, des forces aveugles, qui créent de l'ordre comme la pesanteur fait tomber les corps. Si nous avons pris l'habitude d'identifier ordre et Taxis, arrangement, c'est qu'à nos yeux les phénomènes humains et sociaux sont marqués du sceau de l'intentionnalité au point que nous avons la plus grande peine à reconnaître un ordre qui ne résulte d'aucun dessein, comme le prouvent les résistances rencontrées par Adam Smith quand il a proposé d'attribuer à l'action d'une "main invisible » la façon dont l'homme est conduit à "promouvoir un résultat qui ne faisait nullement partie de ses intentions » (La richesse des nations, citée dans DLL, p. 126). La confusion est d'autant plus grave qu'elle condamne à manquer « le point de départ de la théorie sociale - et sa seule raison d'être - [à savoir] la découverte qu'il existe des structures ordonnées, qui sont le résultat d'hommes nombreux mais d'aucun dessein humain » (DLL 125). Comme on le verra plus loin, la portée de la distinction est encore soulignée par la fait qu'aux deux types d'ordres correspondent deux types d'intervention.

\section{La catallaxie ou ordre spontané du marché.}


Si la notion d'ordre spontané déborde donc largement la sphère des phénomènes sociaux, c'est l'ordre spontané du marché qui intéresse avant tout Hayek. Son approche se caractérise par la prépondérance accordée aux aspects cognitifs du phénomène, le marché étant considéré comme un lieu où s'échangent non pas seulement des marchandises mais des informations. En régime de concurrence, les prix, sur un marché, sont des signaux qui transmettent à chaque agent les connaissances dont il a besoin pour adapter ses décisions à celles des autres, lui permettant ainsi d'utiliser des connaissances qu'à proprement parler il ne possède pas. Cette théorie de la catallaxie restera sans doute la contribution majeure de Hayek à la doctrine économique et toute la question est de savoir si elle suffit à justifier les conclusions qu'il se croit autorisé à en tirer.

Economie et connaissance. Cette dimension cognitive n'est d'ailleurs pas propre à la théorie du marché et s'applique à l'ensemble de la théorie économique. Sur ce point, l'auteur de La route de la servitude se présente comme le continuateur de l'école autrichienne, qui avait en propre d'insister sur le caractère intrinsèquement subjectif des données des sciences sociales. A la différence des sciences naturelles, les données n'y sont pas des faits objectifs, donnés à tous, mais des faits subjectifs, donnés au seul acteur.

Dire en effet que la science économique a pour objet d'étudier des faits «objectifs» est équivoque. Objectifs, ils le sont sans doute, en ce qu'ils forment l'objet d'étude. En ce sens, ils sont donnés à l'économiste, comme le chlore ou le souffre au chimiste; mais il existe une différence profonde entre les deux cas. Alors que le souffre est un corps, ce qui est donné à l'économiste, ce qu'il étudie, ce sont des faits subjectifs, des attentes, des croyances. La monnaie, c'est tout autre chose qu'un bout de métal ou un morceau de papier et, pour la théorie économique, le sucre n'est pas défini par ses qualités objectives, mais " par le fait que les gens croient qu'il servira certains de leurs besoins d'une certaine manière ». On arriverait à la même conclusion si l'on se demandait: pour qui les faits sont-ils supposés établis ? à qui sont-ils donnés : à l'économiste observateur, ou aux personnes dont il veut expliquer l'action? dans ce dernier cas, faut-il supposer que les mêmes faits sont connus de tous, ou faut-il au contraire admettre que les connaissances puissent être très inégalement partagées? Il y a donc deux concepts fondamentalement différents : d'un côté des faits extérieurs, objectifs, supposés connus de l'observateur; de l'autre des chose connues aux personnes dont nous essayons d'expliquer le comportement. D'où le problème du rapport entre ces deux sens : pourquoi les données subjectives devraient-elles correspondre aux données objectives ?

Dans ce contexte, l'apport de Hayek se résume dans l'insistance sur l'aspect social de cette dimension cognitive, qui se manifeste dans la concurrence, le marché, et qui est ainsi intimement lié à la théorie de l'ordre spontané. C'est la volonté de prendre au sérieux ce dernier aspect, de facto absent dans la théorie classique de l'équilibre, qui permet à l'économie de se présenter comme une science authentiquement sociale.

L'essentiel est mis en place dès 1937 dans un article où, après l'auteur lui-même, on s'accorde à reconnaître le cœur de sa théorie économique, et comme le moteur de toute sa pensée.

"A l'évidence, il y a ici [dans la formation de l'équilibre économique] un problème de division de la connaissance, qui est au moins aussi important et assez semblable à celui de la division du travail. Mais alors que cette dernière a constitué un des principaux sujets de recherche depuis le commencement de notre science, la question de la dispersion du savoir a été complètement négligée, bien qu'elle semble constituer le véritable problème de l'économie en tant que science sociale. Le problème que nous prétendons résoudre est celui de la manière dont l'interaction spontanée d'un grand nombre d'individus, chacun d'entre eux possédant seulement des fragments de connaissance, débouche sur un état des affaires dans lequel les prix correspondent aux coûts, etc., et qui pourrait seulement être produit par une direction délibérée de quelqu'un possédant la connaissance combinée de tous ces individus.

[...] La dimension plus large du problème de la connaissance qui attire ici mon attention est [...] la question générale de savoir comment les données subjectives des différentes personnes correspondent aux faits objectifs. Notre problème de connaissance est justement l'existence de cette correspondance qui, la plupart du temps, dans l'analyse de l'équilibre, est simplement supposée 
exister. Mais nous devons pourtant l'expliquer.

[... Ainsi] l'économie s'est approchée beaucoup plus que n'importe quelle autre science sociale d'une réponse à cette question centrale de toutes les sciences sociales: la manière dont la combinaison de fragments de connaissances dispersées dans différents cerveaux individuels peut amener à des résultats qui, s'ils devaient être recherchés délibérément, exigeraient un niveau de connaissance impossible à atteindre par l'esprit dirigeant. Montrer que, dans ce sens, les actions spontanées des individus apporteront, sous des conditions que nous pouvons définir, une distribution de ressources qui peuvent être comprises seulement comme si elles étaient accordées selon un plan unifié, bien que personne ne l'ait planifié, me semble en fait une réponse au problème qui a quelquefois été métaphoriquement décrit comme celui de l' 'esprit social' » [EK, p. 131-133]

Cette part accordée aux considérations cognitives, qui aboutira à voir dans la concurrence une méthode de découverte, _ ou, pour parler comme Foucault, à voir le marché comme lieu de véridiction - , a pris initialement la forme d'une critique de la théorie classique de l'équilibre mais, quel que soit l'intérêt de l'exposé fourni en 1937, il est possible de faire l'économie du passage par la critique des théories classiques et on gagne à suivre une voie où la prise en compte du facteur social est plus directe.

La concurrence comme procédure de découverte. a) Une procédure formelle. La conception classique de la concurrence, la «concurrence parfaite », souffrait entre autre d'être indifférente à l'effet du passage du temps. De là une conception statique, à laquelle Hayek oppose une conception dynamique : La concurrence doit être vue comme un processus, à savoir « un processus dans lequel les gens acquièrent et communiquent de la connaissance » (DLL, p. 733). Dans cette perspective, elle apparaît comme une procédure de découverte, analogue à ce qu'est l'expérimentation dans les sciences de la nature.

La mise en concurrence n'est pas propre à la vie économique. Les concours de recrutement, les compétitions sportives reposent sur le même principe. Il s'agit de savoir qui sera le meilleur. Au point de départ se trouve donc un constat d'ignorance et un besoin de connaissance. Mais dans le cas qui nous intéresse, que veut-on savoir au juste? Il ne suffit pas en effet de reconnaître qu' « aucune théorie ne peut rendre justice [à la concurrence] si les faits à découvrir sont déjà connus » (DLL, p. 732), encore faut-il avoir aussi une idée de ce que l'on cherche. A cet effet, le mieux est d'aller directement au résultat attendu de la catallaxie, à savoir la meilleure allocation possible des ressources disponibles (RTS, p. 32). Les ressources s'entendent ici des ressources matérielles (matières premières, techniques), mais aussi des ressources humaines, i. e. des compétences des agents et en particulier de leurs connaissances relatives aux ressources matérielles; et l'allocation est optimale parce qu'elle est en règle générale la plus efficace, en vertu de son caractère dynamique qui en fait le meilleur moyen de s'adapter à des circonstances toujours changeantes, et de surcroît la seule à respecter les libertés individuelles.

Pour comprendre maintenant comment la concurrence permet de parvenir à ce résultat, il convient de se tourner vers le mécanisme de fixation des prix, et, plus précisément, de considérer ceux-ci sous un jour inhabituel, comme des signaux chargés de transmettre aux agents des informations qui, sans cela, leur resteraient inaccessibles et dont ils ont besoin pour déterminer quelle conduite ils doivent suivre. "La somme d'informations fixée dans les prix est le produit de la concurrence » (DLL, p. 549). Les prix n'ont en effet pas pour seule raison d'être de faciliter les échanges. Ils ont une fonction plus générale, valable pour tous les agents de la vie économique, acheteurs ou non. Ce sont des panneaux indicateurs, qui reflètent, de façon sans doute elliptique, l'état du monde à un instant donné, permettant ainsi à chacun d'étendre sa connaissance au delà de son environnement immédiat. De même, leur variation reflètent les variations de l'état du monde, contribuant ainsi à améliorer la réactivité. De cette façon, le système des prix constitue « un appareil qui enregistre automatiquement les effets des actions individuelles, et dont les indications sont en même temps la résultante et le guide de toutes les décisions individuelles » (RTS, p. 42).

b) Division du travail, coordination et le problème général des sciences sociales. Cette nouvelle approche des prix est au centre de la position défendue par Hayek puisque le refus de 
toute tentative pour modifier de l'extérieur l'ordre spontané du marché en est présenté comme une conséquence directe. L'argument consiste à approfondir la dimension cognitive qui vient d'être introduite et à insister cette fois sur le caractère éclaté de cette connaissance. La division du travail est aussi une division du savoir. Le caractère imparfait de la concurrence tient à ce que, hormis dans les cas les plus simples, aucun agent ne dispose d'une connaissance parfaite, qu'il n'y a pas d'omniscience. L'imperfection est due à la complexité des phénomènes et croît avec elle.

Considérer les prix dans leur dimension cognitive, comme des signaux chargés de transmettre des informations, présuppose que les acteurs ne disposent pas tous en même temps de toute l'information, en d'autres termes, que celle-ci est divisée puisque, si ce n'était pas le cas, si tout le monde disposait de la même information, les prix perdraient aussitôt la fonction qui leur est attribuée. L'éclatement du savoir, l'ignorance de données essentielles apparaissent ainsi comme la donnée fondamentale.

Le problème à résoudre apparaît cette fois comme un problème de coordination, c'est-à-dire comme un cas particulier d'un problème général posé par la division du travail, quand celle-ci prend la forme de la division du savoir. Pris isolément, «tout seul dans son coin », aucun individu ne peut trouver la meilleure allocation possible des ressources. Une fois introduite l'idée de coordination, la complexité des facteurs à coordonner intervient de façon cruciale, puisque c'est uniquement pour les phénomènes complexes que l'ordre du marché se trouve offrir la meilleure coordination possible. Dans les cas simples la solution, elle aussi, est simple, c'est-à-dire à la portée d'un individu isolé, de sorte que le problème de coordination disparaît. C'est quand il devient impossible à un individu isolé de prendre une vue synoptique de la situation que la décentralisation s'impose; et c'est la décentralisation qui fait surgir le problème de la coordination.

«C'est la complexité même de la division du travail à l'époque moderne qui fait de la concurrence la seule méthode susceptible de réaliser la coordination recherchée [...] La décentralisation étant devenue nécessaire parce que personne ne peut consciemment équilibrer toutes les considérations relatives aux décisions d'un si grand nombre d'individus, il est clair que la coordination ne saurait être atteinte par un «contrôle conscient», mais uniquement par des dispositifs qui transmettent à chaque agent d'exécution les renseignements dont il a besoin pour adapter efficacement ses décisions à celles des autres. Et comme aucun centre ne saurait connaître complètement ni rassembler et disposer assez vite tous les détails des modifications qui ne cessent d'affecter l'offre et la demande des divers produits, on a besoin d'un appareil qui enregistre automatiquement les effets des actions individuelles, et dont les indications sont en même temps la résultante et le guide de toutes les décisions individuelles.

C'est là précisément ce que fait le système des prix en régime de concurrence. [...] Plus l'ensemble est compliqué, plus nous dépendons de cette division de la connaissance entre individus dont les efforts isolés sont coordonnés par un mécanisme impersonnel de transmission des renseignements : ce mécanisme, nous l'appelons le système des prix » (RTS, p. 42).

La valeur irremplaçable du marché, de la concurrence, vient donc de ce qu'ils offrent une solution simple et inattendue à un problème qu'on pouvait croire insoluble : coordonner sans que personne ne coordonne. C'est presque un miracle, impensable sans l'intervention d'une main invisible. Il convient en effet de ne pas oublier que d'ordinaire les problèmes de ce type sont résolus d'une autre façon. Sur un chantier, il y a un chef de chantier qui coordonne l'action des différents corps de métier; dans un orchestre, il y a un chef d'orchestre qui coordonne l'action des différents musiciens. La coordination est assurée par un coordinateur et, en présence d'un ordre, nous sommes naturellement portés à l'attribuer à un ordonnateur. D'où l'originalité des ordres spontanés, et notre difficulté à en reconnaître l'existence. Ceci étant établi, il ne reste plus, pour compléter l'argument, qu'à montrer que l'ordre spontané du marché est nécessairement supérieur à un contrôle délibéré et à comparer ce dernier type d'intervention avec celui proposé par Hayek pour assurer la bonne marche des ordres spontanés.

Les conditions de possibilité des catallaxies, ou théorie de l’intervention. 
Dans le cas des interventions à proscrire, il suffira de retenir qu'il convient d'y distinguer deux aspects : la critique du planisme d'une part et le refus plus général de toute tentative de régulation exogène directe d'autre part. Le plus instructif se trouve en effet dans le rapport étroit établi entre l'ordre spontané du marché et un certain état du droit. Hayek est si peu hostile à l'intervention de l'Etat qu'il la juge doublement indispensable, quitte à la nommer autrement. Tout d'abord, il est demandé à celui-ci de veiller à ce que rien ne vienne entraver le libre exercice des forces ordonnatrices; semblable en cela au service d'entretien dans une firme, il lui incombe de s'assurer qu'une catallaxie est en bon état de marche. Mais un ordre spontané ne peut surgir que dans des circonstances spécifiques et l'Etat doit également imposer aux individus les règles juridiques de conduite qui conditionnent l'existence de l'ordre spontané. Dans le premier cas, ôter toute entrave peut prendre deux formes opposées. La « déréglementation » à laquelle on pense immédiatement ne suffit pas. Il se trouve en effet que l'autorégulation du marché comporte des failles et que cet ordre spontané produit spontanément une tendance à la concentration et au monopole qui menace de le détruire. Pour que la concurrence continue à jouer son rôle il y a donc lieu de défendre en quelque sorte le marché contre lui-même en imposant des lois anti-trusts (Principes d'un ordre social libéral, § 52-55, dans SPPE p. 267-268).

Ordre économique et ordre juridique. Alors qu'il ne s'agit là que de mesures ponctuelles, le second type d'action, qui englobe le premier, correspond, lui, à une des intuitions fondamentales de Hayek : " dans un ordre économique comportant une division du travail très poussée, il ne peut plus s'agir de poursuivre des objectifs communs concrètement perçus mais il faut se guider sur des règles abstraites de conduite, et il $\mathrm{y}$ a un lien étroit entre ces règles et la formation de l'ordre économique » (DLL 896-97). L'importance de ces considérations, qui font dépendre le bon fonctionnement du marché d'un certain type de droit et que Hayek résume dans la notion de rule of law (Etat de droit, Rechtsstaat), vient de ce qu'elles ont une portée beaucoup plus générale. Avec elles, on sort de la sphère purement économique pour passer dans la sphère juridique, qui vaut pour tous et embrasse l'ensemble de la vie sociale. Le point avait été très clairement formulé par Louis Rougier dans son allocution au colloque Lippmann de 1938 :

le régime libéral n'est pas seulement le résultat d'un ordre naturel spontané [...] il est aussi le résultat d'un ordre légal qui suppose un interventionnisme juridique de l'Etat. La vie économique se déroule dans un cadre juridique qui fixe le régime de la propriété, des contrats, des brevets d'invention, de la faillite, le statut des associations professionnelles et des sociétés commerciales, la monnaie et la banque, toutes choses qui ne sont pas des données de la nature, comme les lois de l'équilibre économique, mais des créations contingentes du législateur. Il n'y a dès lors aucune raison de supposer que les institutions légales, historiquement existantes à l'heure actuelle, soient d'une façon définitive et permanente les mieux appropriées à la liberté des transactions. La question du cadre légal le mieux approprié au fonctionnement le plus souple, le plus efficace, le plus loyal du marché a été négligée par les économistes classiques. (Cité dans Foucault, p. 166-67).

Autant dire que l'ordre spontané ne suffit pas à fonder le libéralisme. Ce n'est qu'un des deux piliers sur lesquels il repose et, faute d'un droit qui soit là pour l'imposer, cet ordre spontané serait exposé à toute sorte de perturbations, qui risqueraient de compromettre jusqu'à son existence.

Ce rôle constitutif du droit mis en évidence, reste à en préciser le mode de fonctionnement : il consiste non en commandements spécifiques, mais en règles générales de conduite, qui opèrent sur les forces ordonnatrices. C'est dans ce cadre qu'il faut situer le contraste déjà signalé entre abstrait et concret : la règle est générale, abstraite, par opposition au commandement, particulier, concret. Derrière le reproche d'omniscience adressé au planiste, ce qui est visé, c'est une attention excessive au concret, la volonté de tout régler jusque dans les moindres détails. Le type de « règle » qui y correspond est le commandement, qui s'adresse à un individu particulier et n'est précisément pas une règle, alors que la loi, entendue comme règle de juste conduite, est universelle et abstraite : elle doit s'appliquer également à tous et se doit pour cela de faire abstraction des individus. Pour régler la circulation, une solution consisterait à dire à chacun où il doit aller mais on préfère d'ordinaire établir un code de la route. 
Le caractère abstrait d'une règle, et de l'ordre qui en résulte, explique la plupart des propriétés des ordres spontanés. Ainsi, c'est ce qui explique que, au lieu d'être reconnus comme ordonnés, ils soient souvent pris pour des désordres injustifiables. C'est encore ce qui explique les difficultés que les phénomènes sociaux opposent à la prédiction. D'un côté, il est impossible de prédire l'effet des lois, de savoir par exemple qui elles avantageront et, à supposer que ce soit possible, il faut s'interdire de le faire. D'un autre côté, elles doivent nous permettre de prédire le comportement des individus, ce qui est indispensable si nous devons pouvoir faire des projets. Ces propriétés en apparence incompatibles découlent toutes deux de la nature abstraite de la loi et s'expliquent par la distance qui sépare la conduite dictée par la règle de ses résultats, considérés ici comme seuls concrets, distance qui se retrouve dans le caractère inintentionnel des ordres spontanés. Ainsi encore le type de contrôle que nous pouvons exercer sur les ordres spontanés dans un cas, sur les ordres fabriqués dans l'autre, renvoie à l'opposition entre règles abstraites de bonne conduite et commandements concrets (DLL, p. 132).

$* \quad *$

$\mathrm{Si}$, dans ses grands traits, la théorie des ordres spontanés a été élaborée dès avant 1940 pour ne plus guère varier par la suite, elle a donné lieu à des présentations assez différentes, selon qu'il s'agissait de lutter contre le planisme un temps menaçant ou au contraire de formuler la doctrine d'un néo libéralisme en passe de triompher. Il n'est pourtant pas nécessaire d'entrer dans tous les détails pour porter un premier jugement sur les arguments avancés et reconnaître que, quels qu'en soient les mérites intrinsèques, la théorie des prix du savant autrichien n'autorise pas certaines des conclusions qu'il croit pouvoir en tirer. Le problème le plus immédiatement identifiable est le pan-économisme, qui pose la validité universelle de l'analyse en termes de marché et conduit en particulier à inverser le rapport traditionnellement établi entre économie et gouvernement. Toutefois, au plan conceptuel, il y a lieu de se demander si la confusion établie entre les deux couples naturel-conventionnel et naturel-artificiel n'est pas beaucoup plus grave, dans la mesure où elle affecte directement la notion d'ordre spontané.

Un des traits les plus remarquables du néo-libéralisme est sans conteste la façon dont il a étendu l'analyse économique à des domaines considérés jusqu'alors comme ne relevant pas de sa compétence. C'est ainsi par exemple que Gary Becker a interprété l'éducation, ou le système pénitentiaire en termes de marché ; s'est même développée une "market biology », proposant d'appliquer cette même grille d'analyse à l'étude du comportement animal. Si de telles extensions n'ont pas été le fait d'Hayek lui-même, il en avait posé les principes, puisqu'à ses yeux « l'économiste est fondé à souligner que pour juger de toutes les institutions particulières, l'on doit accepter comme critère leur aptitude à favoriser l'existence et l'amélioration de cet ordre [économique spontané]»(DLL, p. 542). Dans ce cadre, mention spéciale doit être faite de ce qui concerne le rapport de l'économie à la politique. Il a été établi plus haut que l'ordre spontané du marché ne pouvait fonctionner en dehors d'un cadre juridique approprié. Il ne suffit cependant pas que le gouvernement soit requis d'utiliser la force contraignante du droit pour assurer «la préservation d'un ordre de marché efficient»(SPPE, p. 267). Les institutions politiques elles-mêmes sont redevables de l'analyse économique, de sorte qu'au terme, on a « un Etat sous surveillance du marché plutôt qu'un marché sous surveillance de l'Etat ».

Le plus grave est cependant ailleurs, dans l'identification erronée établie entre le couple naturel-artificiel et le couple naturel-conventionnel (ou positif: phusei/thesei), qui induit une approche biaisée de notre pouvoir d'intervention sur l'ordre spontané entendu dans toute sa généralité.

A en croire Hayek, une des raisons pour lesquelles nous aurions tant de peine à reconnaître l'existence des ordres spontanés serait à chercher dans la « distinction mal venue introduite par les Grecs », entre phénomènes naturels et phénomènes artificiels, distinction qu'il présente comme équivalente à celle introduite par les sophistes entre naturel et positif (DLL, p. 90-91; cf. p. 920 n et SPPE p. 160-161). Rien n'autorise pourtant une telle identification, ni historiquement ni 
conceptuellement. Les deux distinctions n'ont ni le même lieu ni le même mode de fonctionnement. La dernière s'applique au droit (droit naturel-positif) et aux institutions. La première appartient à un tout autre domaine et caractérise deux types de production : l'art d'où procède l'artificiel, c'est la technè aristotélicienne. Alors que le positif et le naturel n'ont aucun rapport entre eux, l'artificiel présuppose le naturel, qu'il ne fait que modifier et en quelque sorte prolonger. Le conventionnel est intrinsèquement social (peut-on passer une convention avec soi-même ??) : il faut deux parties qui s'accordent ; corrélativement il n'est nullement besoin de se rapporter à la nature. Inversement le rapport à la nature est constitutif de l'idée de technique. L'art, a-t-on dit, c'est l'homme ajouté à la nature ; rien cette fois d'intrinsèquement social, Robinson sur son île travaillait seul et pour lui seul.

Or, il est assez clair que Hayek glisse sans cesse d'un couple à l'autre. D'un côté, dans l'opposition entre Kosmos et Taxis, c'est le premier qui est en jeu : la disposition des pièces sur l'échiquier est une convention et on hésitera à y voir quelque chose d'artificiel, faute d'une disposition naturelle à lui opposer; de même, le commandement, associé à la Taxis, n'a pas de place dans la technique, sauf à parler d'un commandement fait à la nature. D'un autre côté cependant, lorsque, dans The Counter-Revolution of Science, Hayek dénonce l'hybris polytechnicienne et le scientisme des ingénieurs qui veulent appliquer aux phénomènes sociaux les méthodes qui ont fait leur preuve dans l'étude des phénomènes naturels sans se demander si cette extension est légitime, ce qui est en cause cette fois c'est le contraste entre le naturel et l'artificiel. On est donc en droit de retourner l'objection : Hayek ne voit pas que l'idée d'ordre spontané a une extension beaucoup plus grande que celle qu'il lui reconnait. C'est l'ordre du monde tout entier qui est spontané, comme la chute libre est là pour nous le rappeler. S'il est vrai que la nature est l'ensemble des phénomènes en tant qu'ils sont soumis à des lois, les phénomènes sociaux, dès lors qu'on les reconnaît soumis à des lois, deviennent des phénomènes naturels. Rien n'autorise à revendiquer en leur faveur un statut d'exception. Le dualisme hérité de Carl Menger induit une lacune dans la conception de l'ordre. Dès lors que l'on considère l'ordre naturel dans son ensemble il devient clair qu'il est tout autant modifiable, perfectible, que spontané. Sans doute, il y a bien, par le biais de ce qu'il dit des interventions, une théorie de l'ordre modifiable chez Hayek. Mais elle est comme extérieure à la théorie de l'ordre et, dans ce domaine, on est donc fondé à considérer la pensée de Hayek comme lacunaire : l'ordre doit être pensé non seulement comme spontané, mais aussi comme modifiable et le second qualificatif est tout aussi important que le premier. Hayek se plaignait qu'on ait du mal à reconnaître l'existence d'ordre spontané ; son œuvre prouve qu'on a encore plus de mal à reconnaitre celle d'un ordre modifiable. 